\title{
Effects of Intense Laser Pulse Properties on Wake Field Acceleration in Magnetized Plasma: Half-Sine Shape (HSS) and Gaussian Shape (GS) Pulses
}

\author{
Amir Rahimian and Hossein Zahed \\ Department of Physics, Sahand University of Technology, Tabriz, Iran \\ Corresponding Author's Email: zahed@sut.ac.ir \\ Received: Nov. 13, 2017, Revised: Mar. 28, 2018, Accepted: Apr. 30, 2018, Available Online: Dec. 27,2019 \\ DOI: 10.29252/ijop.13.2.111
}

\begin{abstract}
In this paper, we have simulated the excitation of wake fields in the interaction of an intensive laser pulses having Half-Sine and Gaussian time envelopes with a fully ionized cold plasma using particle in cell (PIC) method. We investigated the dependency of wake filed amplitude to different laser and plasma parameters such as laser wavelength, pulse duration and electron number density. In addition, the effect of employing a longitudinal magnetic field on the intensity of wake field is studied. It has been seen that the wake field intensity is enhanced in the presence of a magnetic field for both Half-Sine and Gaussian shape pulses. Our aim has been finding optimum values of different parameters for which higher accelerating wake electric fields can be obtained.
\end{abstract}

KEYWORDS: laser pulse, wake field excitation, particle in cell (PIC), magnetized plasma

\section{I.INTRODUCTION}

Laser plasma interactions have become an interesting subject since the time they started to have higher intensities about a few decades ago. It has different applications such as laser driven fusion, plasma based accelerators and $\mathrm{X}$-ray lasers [1-3]. A lot of struggles have been made to attain high accelerating electric fields in an under dense plasma by employing laser pulses with ultrahigh intensities and short wavelengths. There is a great deal of applications of accelerated charged particles in physical sciences. Plasma based accelerators have several schemes in which the working principal is the same: laser wake field accelerator (LWFA) [4], plasma wake field accelerator (PWFA) [5], plasma beat wave accelerator (PBWA) [6-8], self-modulated laser wake field accelerator (SM-LWFA) [911] and wake field accelerators driven by multiple electron or laser pulses [12,13]. The LWFA was introduced before other schemes. After introducing the LWFA idea [4], a noteworthy advancement has been made in both experimental [14-16] and theoretical physics [17]. However, there are a lot of efforts to be made to put all physical burdens away from reaching future high gradient accelerators. One of the most important subjects is to control the charge and energy of the electron beam and improve its quality. Many nonlinear effects are present in the process of electron acceleration via laser plasma interactions, such as fast electron and ion generation [18,19]. Magnetic fields have an incontrovertible effect on nonlinear plasma dynamics. A jet of fast electrons flowing along the laser propagation direction [20] or a nonlinear current of the plasma electrons [21] is usually used to generate magnetic fields. Generation of magnetic fields up to $70 T$ using the technology of pulsed magnets is also reported [22]. Intense magnetic fields have expressive effects on particle transport, propagation of laser pulses and laser beam self-focusing. Shukla et al. have studied the nonlinear propagation of an electromagnetic wave in strongly magnetized electronpositron-ion plasma. They have observed that the generation of the wake fields depends on the presence of the ion species and the external 
magnetic field [23]. Krasovitskii et al. have studied the interaction of powerful laser pulse with plasma and generated wake field in the presence of an external magnetic field [24]. They have found that for various angles between the direction of laser pulse propagation and magnetic field, the transferred energy to electron oscillations in the wake field is different.

It is evident that applying an external magnetic field has complicated effects on laser plasma nonlinear processes. So, various features of this issue still need to be studied. Numerical simulation is an important and efficient tool in investigations. The code to model a plasmabased accelerator should be able to simulate the acceleration of particles, the wake generation and the driver evolution. Among a variety of methods, particle based models can do this appropriately because the trajectories of particles should be followed in their selfconsistent fields. The most reliable particle based model is the fully explicit particle in cell (PIC) method [25]. It simulates the interaction dynamics of charged particles with electromagnetic fields self-consistently using a grid of one, two or three dimensions [26].

\section{The BASIC EQUATIONS AND AlgorithM}

In this paper, three-dimensional particle in cell simulations are carried out to study the wake field produced by propagation of an intense linearly polarized laser pulse with a transverse Gaussian profile and two different temporal envelopes (Figs. 1(a-b)): Gaussian shape (GS) and Half-Sine shape(HSS).

We have also studied the effect of the presence of a magnetic field along the laser propagation direction on the wake field excitation. The ambient plasma is homogeneous, cold and preionized $\left(\mathrm{He}^{++}\right)$with a linear density ramp of the form $n(x)=2 a x+b \quad$ where $n(0)=n^{1}, n(L)=n^{2}$ and $L$ is ramp length. The driving laser beam is in TM-mode (Spolarized) with a wavelength of $\lambda=0.8 \mu \mathrm{m}$ corresponding to a frequency of
$\omega_{L}=2 \pi c / \lambda=2.36 \times 10^{15} \mathrm{~Hz}$, where $c$ is the light speed in vacuum. The plasma bulk density $\left(n_{0}\right)$ is $5 \times 10^{24} \mathrm{~m}^{-3}$ which corresponds to the plasma wavelength of $15 \mu \mathrm{m}$ via $\lambda_{P}(\mu \mathrm{m})=3.3 \times 10^{10} / \sqrt{n_{0}\left(\mathrm{~cm}^{-3}\right)}$.

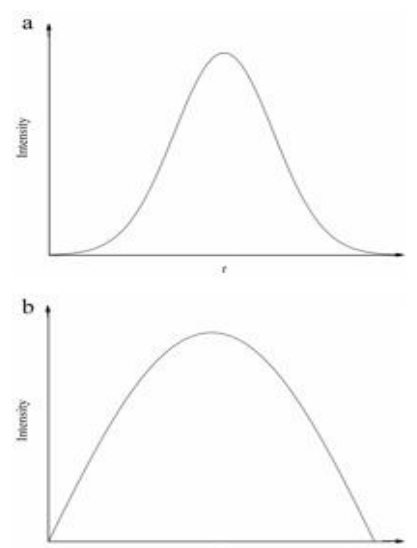

Fig. 1. Profiles of laser pulse: (a) Gaussian shape and (b) Half-Sine shape.

The ions are assumed to be stationary forming a uniform background of charges. The intensity of laser pulse employed in this simulation is $I_{0}=3 \times 10^{22} \mathrm{~W} / \mathrm{m}^{2}$ which falls into relativistic regime. Laser pulse intensity is related to the dimensionless laser strength parameter $a_{0} \quad$ by $a_{0}=0.85 \lambda(\mu \mathrm{m}) \sqrt{I\left(10^{22} \mathrm{~W} / \mathrm{m}^{2}\right)}$. So, the above $I_{0}$ and $\lambda$ values yield $a_{0}=1.18$. The electron motion is highly relativistic when $a_{0} \gtrsim 1$. This relativistic effect sets the stage for selffocusing [27]. The general expression for the index of refraction in a plasma is given by $\eta_{r}=1-\frac{\omega_{p}}{2 \omega_{L}^{2}} \frac{n(r)}{n_{0} \gamma(r)}, \quad$ where $\gamma(r)$ is the relativistic factor, $n(r)$ is radial density (Cylindrical) profile, $\omega_{P}$ is the plasma electron frequency prior to the passage of laser pulse: $\omega_{P}=\left(4 \pi n_{0} e^{2} / m_{e}\right)^{1 / 2} \quad$ and $\quad \gamma=\gamma_{\perp}=\left(1+a^{2}\right)^{1 / 2}$. Here, $a^{2}=\mathbf{a} \cdot \mathbf{a}$ where $\mathbf{a}$ is the normalized vector potential of the laser field: $\mathbf{a}=e \mathbf{A} / m_{e} c^{2}$ where $e$ is the fundamental charge and $m_{e}$ is the electron mass. A laser intensity profile peaked on axis $\frac{\partial a^{2}}{\partial r}<0$ leads to $\frac{\partial \eta_{r}}{\partial r}<0$ which 
implies that the phase velocity along the propagation axis is less than it is off-axis. This causes the laser pulse fronts to curve such that the beam focuses towards the axis. The mathematical expressions for laser pulses are $a=a_{0} \exp \left(-r^{2} / s_{0}^{2}\right) \exp \left(-t^{2} / \tau^{2}\right) \quad$ (GS) and $a=a_{0} \exp \left(-r^{2} / s_{0}^{2}\right) \cos \left[k_{p}(x-c t)\right]$ (HSS). Other considered laser parameters are full width at half maximum (FWHM) of $9 \mu \mathrm{m}$ (or laser pulse duration of $\tau=30 \times 10^{-15} \mathrm{~s}$ ) and spot size of $s_{0}=15 \mu \mathrm{m}$. In this simulation we consider a plasma box with the grid size of $(\mathrm{Nx}, \mathrm{Ny})=(750,256)$ equal to $60 \mu m x$-direction length and $204.8 \mu \mathrm{m} y$-direction length. Each cell contains 6 particles. The time step meets Courant-Levy stability criterion $\Delta t \leq \frac{1}{c}\left(\sum_{i} \frac{1}{\left(\Delta x_{i}\right)^{2}}\right)^{-1 / 2}$ and the program runs for 1500 time steps.

The laser pulse is launched from left boundary and propagates along $x$-direction. Other boundaries are considered to be conductors.

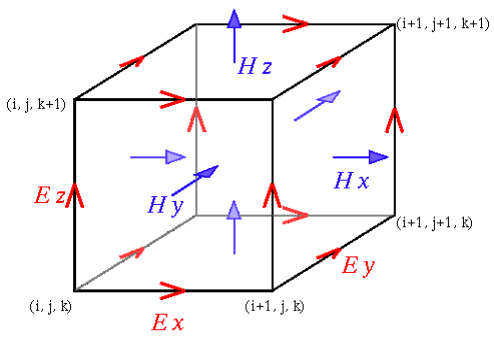

Fig. 2. Yee mesh

We used the conventional PIC algorithm in our simulation which is briefly described in the following. First, the simulation domain is divided into spatial grids using Cartesian geometry. Then, the plasma particles are loaded onto the grids and their charges are assigned to the surrounding nodes (using firstorder weighting) from which currents are calculated. These source terms are employed to obtain the electric and magnetic fields via Maxwell equations (SI) [28]:

$\nabla \times \mathbf{E}=-\frac{\partial \mathbf{B}}{\partial t}$ $\nabla \times \mathbf{B}=\mu_{0} \varepsilon_{0} \frac{\partial \mathbf{E}}{\partial t}+\mu_{0} \mathbf{J}$

where the Finite-Difference Time-Domain (FDTD) method is used to solve Maxwell equations. Also, in order to make sure that continuity equation $\left(\frac{\partial \rho}{\partial t}+\nabla \cdot \mathbf{J}=0\right)$ is observed, current density $\mathbf{J}$ is calculated using a charge-conservation scheme (CCS). Then, to discretize $\mathbf{E}$ and $\mathbf{B}$ fields, they are defined on the Yee-mesh (Fig. 2) [29].

For a uniform 3D Cartesian discretization of the fields (the grid spacings are $\Delta x, \Delta y, \Delta z$ ), the FDTD scheme can be therefore written explicitly as:

$$
\begin{aligned}
& \frac{B_{x}^{t+1 / 2}\left(i, j+\frac{1}{2}, k+\frac{1}{2}\right)-B_{x}^{t-1 / 2}\left(i, j+\frac{1}{2}, k+\frac{1}{2}\right)}{\Delta t}= \\
& \frac{E_{y}^{t}\left(i, j+\frac{1}{2}, k+1\right)-E_{y}^{t}\left(i, j+\frac{1}{2}, k\right)}{\Delta z} \\
& -\frac{E_{z}^{t}\left(i, j+1, k+\frac{1}{2}\right)-E_{z}^{t}\left(i, j, k+\frac{1}{2}\right)}{\Delta y} \\
& \mu_{0} \varepsilon_{0} \frac{E_{x}^{t}\left(i+\frac{1}{2}, j, k\right)-E_{x}^{t-1}\left(i+\frac{1}{2}, j, k\right)}{\Delta t}= \\
& \frac{B_{z}^{t-1 / 2}\left(i+\frac{1}{2}, j+\frac{1}{2}, k\right)-B_{z}^{t-1 / 2}\left(i+\frac{1}{2}, j-\frac{1}{2}, k\right)}{\Delta y} \\
& -\frac{B_{y}^{t-1 / 2}\left(i+\frac{1}{2}, j, k+\frac{1}{2}\right)-B_{y}^{t-1 / 2}\left(i+\frac{1}{2}, j, k-\frac{1}{2}\right)}{\Delta z} \\
& +\mu_{0} J_{x}^{t-1 / 2}\left(i+\frac{1}{2}, j, k\right)
\end{aligned}
$$

where a grid point of the space is denoted as $(i, j, k) \equiv(i \Delta x, j \Delta y, k \Delta z)$. The equations for $E_{y}$, $E_{z}, B_{y}$, and $B_{z}$ can be similarly constructed. Thereafter, the updated $\mathbf{E}$ and $\mathbf{B}$ fields are used to push the particles into new positions and velocities by employing relativistic Lorentz equation of motion:

$\frac{d \mathbf{P}}{d t}=q\left(\mathbf{E}+\frac{\mathbf{P}}{\gamma} \times \mathbf{B}\right)$

$\mathbf{P}=\gamma \mathbf{V}$ is the relativistic particle momentum where $\gamma$ is the relativistic factor. Here, Boris scheme is used [30] which is comprised of 
three steps: half acceleration, rotation and half acceleration. The difference form of Eq. (3) is:

$\frac{\mathbf{P}^{t+1 / 2}-\mathbf{P}^{t-1 / 2}}{\Delta t}=q\left(\mathbf{E}^{t}+\frac{\mathbf{P}^{t+1 / 2}+\mathbf{P}^{t-1 / 2}}{2 \gamma^{t}} \times \mathbf{B}^{t}\right)$

$\mathbf{P}^{-}=\mathbf{P}^{t-1 / 2}-q \mathbf{E}^{t} \frac{\Delta t}{2}($ half acceleration $)$

$\mathbf{P}^{+}=\left(\begin{array}{ccc}1-S_{z} T_{z}-S_{y} T_{y} & S_{y} T_{x}+S_{z} & S_{z} T_{x}-S_{y} \\ S_{x} T_{y}-S_{z} & 1-S_{x} T_{x}-S_{z} T_{z} & S_{z} T_{y}+S_{x} \\ S_{x} T_{z}+S_{y} & S_{y} T_{z}-S_{x} & 1-S_{x} T_{x}-S_{y} T_{y}\end{array}\right) \mathbf{P}^{-}$

(rotation)

$\mathbf{P}^{t+1 / 2}=\mathbf{P}^{+}+q \mathbf{E}^{t} \frac{\Delta t}{2}($ half acceleration $)$

where

$\mathbf{T}=\frac{\Delta t}{2 \gamma^{t}} \mathbf{B}^{t}, \mathbf{S}=\frac{2 \mathbf{T}}{1+T^{2}}$ and $\gamma^{t}=\sqrt{1+P^{ \pm^{2}}}$

These new momentums of particles are used to advance positions $(\mathbf{r} \equiv x, y, z)$ :

$\frac{\mathbf{r}^{t+1}-\mathbf{r}^{t}}{\Delta t}=\frac{\mathbf{P}^{t+1 / 2}}{\gamma^{t+1 / 2}}$

This cycle is repeated for as many time steps as required.

\section{III.EFFECT OF ELECTRON NUMBER DENSITY}

Electron number density plays an incontrovertible role in laser plasma interaction. It affects the properties of wake field significantly. In order to study the effect of this parameter, the amplitude of wake field, $E_{w f}$, is plotted in terms of $n_{0}$ in Figs. 3(a-f) for both envelop shapes and three different laser wavelengths: $\lambda=0.8 \mu \mathrm{m}, 1.1 \mu \mathrm{m}$, and $1.4 \mu \mathrm{m}$, respectively. It can be seen from Fig. 3 that as $n_{0}$ increases, $E_{w f}$ grows steeply until its resonance value is reached. Thereafter, it drops gradually. However, the behavior of $E_{w f}$ is different in the studied range of $n_{0}$ for $\lambda=1.4$ $\mu m$ case and shows an upward trend. Also, longer laser wavelengths lead to higher $E_{w f}$ amplitudes. This behavior is in complete agreement with Fig. 1(b) in Askari and Shahidani [31]. The maximum amplitude of wake field for different $\lambda$ values are shown in Table 1. When the magnetic field is applied, the curves shift towards higher intensities. Both HSS and GS have the largest upshift in $E_{w f}$ for $\lambda=1.1 \mu \mathrm{m}$.

\section{IV.EfFect of LASER PUlSe DURATION}

Another important parameter governing wake field excitation is the laser pulse duration. So, $E_{w f}$ in terms of $\tau$ is shown in Figs. 4a-4f for $\lambda=0.8 \mu \mathrm{m}, 1.0 \mu \mathrm{m}$ and $1.2 \mu \mathrm{m}$, respectively. It is observed that for these three wavelengths, $E_{w f}$ peaks at $c \tau \approx \lambda_{p}, c \tau \approx 0.9 \lambda_{p}$ and $c \tau \approx 0.8 \lambda_{p}$ for HSS and at $c \tau \approx 0.8 \lambda_{p}, c \tau \approx 0.7 \lambda_{p}$ and $c \tau \approx 0.6 \lambda_{p}$ for GS, respectively. This trend exhibits that an increase in $\lambda$ gives rise to a decrease in laser pulse length $(c \tau)$ at which $E_{w f}$ is maximum. In other words, more energy is transferred from laser pulse to wake field when shorter laser pulse lengths are employed. The behavior of $E_{w f}$ in this case is the same as Fig. 3b in Askari and Shahidani [31]. However, the effect of different $\lambda$ values is not studied in Askari and Shahidani [31]. Moreover, pulse lengths at which $E_{w f}$ peaks are shorter for GS compared to HSS. The maximum amplitude of wake field for different $\lambda$ values are shown in Table 2 . In the presence of magnetic field, $E_{w f}$ grows obviously and the curves shift up for both HSS and GS.

\section{EFFECT OF ENVELOPE SHAPE}

In order to study how different laser pulse shapes influence wake field excitation, we compared HSS and GS pulses. The simulation was done in the presence and absence of magnetic field, but the results are shown only in propagation direction. In Figs. 5(a-b), the amplitude of wake field in terms of propagation coordinate $x$ is plotted for $B_{x}=0$ and $60 T$, respectively. It is seen clearly that GS pulse generates larger accelerating wake fields in comparison with HSS pulse. This case is not studied by Askari and Shahidani [31]. Applying magnetic field causes the magnitude of $E_{w f}$ to rise for both envelope shapes, but GS undergoes more growth than HSS. 

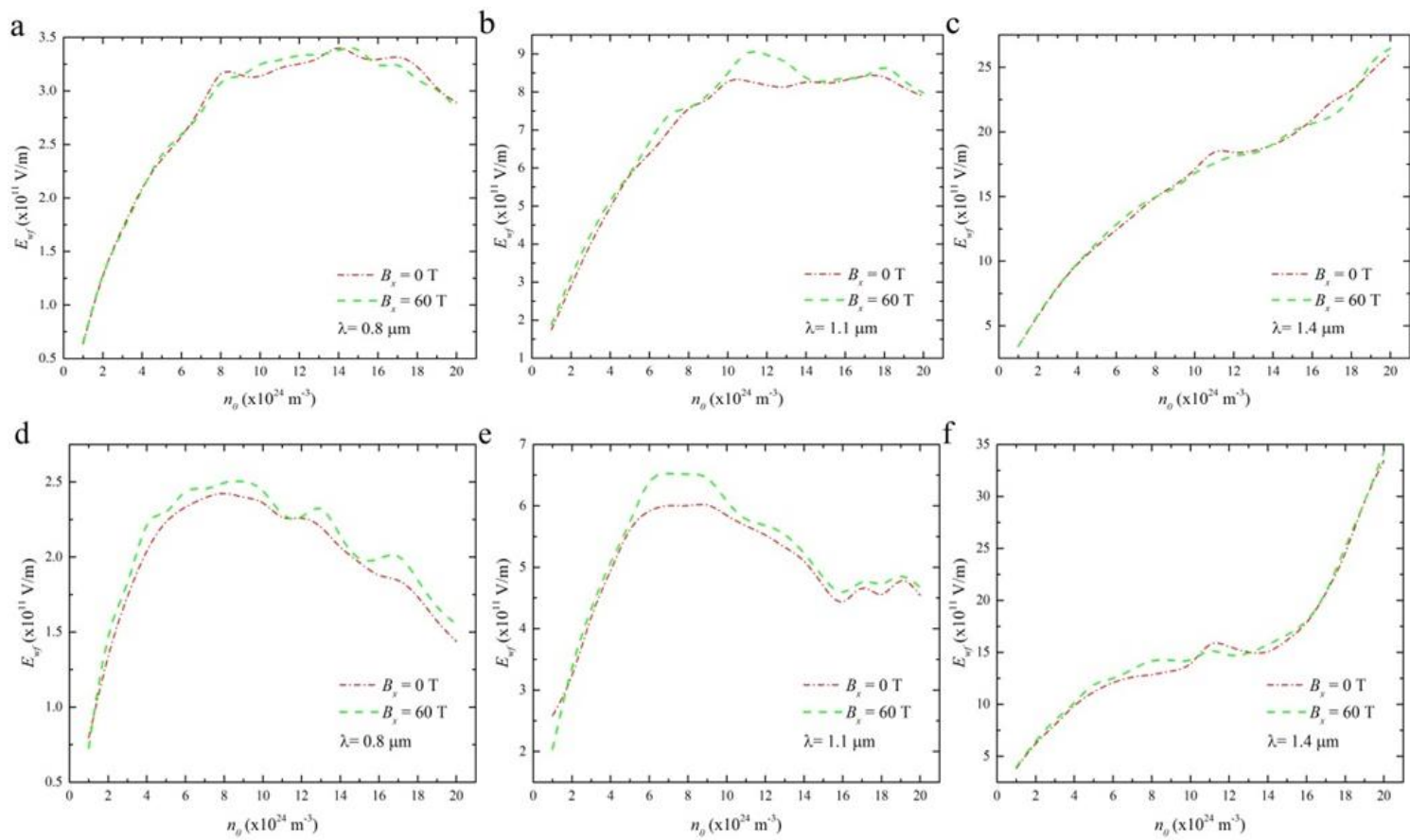

Fig. 3. Amplitude of wake field $E_{w f}$ vs. $n_{0}$ for three different wavelengths for HSS (a-c) and (f) GS (d-f).

Table 1. Maximum amplitude of wake field in terms of $n_{0}$ for three different wavelengths.

\begin{tabular}{|ccccc|}
\hline $\begin{array}{c}\text { Wavelength } \\
(\mu \mathrm{m})\end{array}$ & $\begin{array}{c}E_{w f}^{\max }(\mathrm{HSS}) \\
\left(\times 10^{11} \mathrm{~V} / \mathrm{m}\right)\end{array}$ & $\begin{array}{c}n_{0} \\
\left(\times 10^{24} \mathrm{~m}^{-3}\right)\end{array}$ & $\begin{array}{c}\mathrm{E}_{\text {wf }}^{\max }(\mathrm{GS}) \\
\left(\times 10^{11} \mathrm{~V} / \mathrm{m}\right)\end{array}$ & $\begin{array}{c}n_{0} \\
\left(\times 10^{24} \mathrm{~m}^{-3}\right)\end{array}$ \\
\hline 0.8 & 3.454 & 14 & 2.437 & 8 \\
\hline 1.1 & 8.455 & 18 & 6.067 & 9 \\
\hline 1.4 & 26.044 & 20 & 33.456 & 20 \\
\hline
\end{tabular}
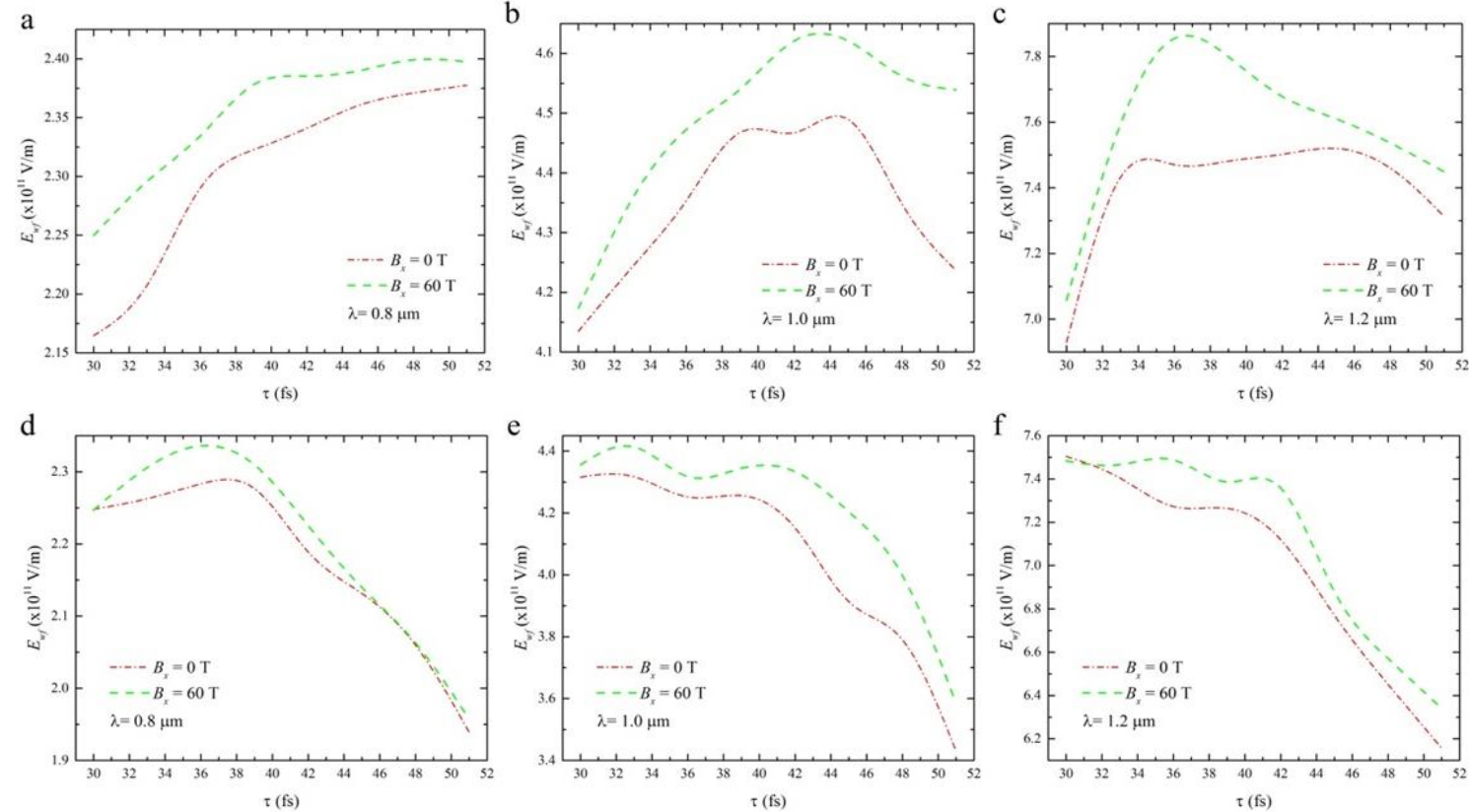

Fig. 4. Amplitude of wake field $E_{w f}$ vs. $\tau$ for three different wavelengths for HSS (a-c) and (f) GS (d-f)

Table 2. Maximum amplitude of wake field in terms of $\tau$ for three different wavelengths

\begin{tabular}{|ccccc|}
\hline $\begin{array}{c}\text { Wavelength } \\
(\mu \mathrm{m})\end{array}$ & $\begin{array}{c}\mathrm{E}_{\mathrm{wff}}^{\max }(\mathrm{HSS}) \\
\left(\times 10^{11} \mathrm{~V} / \mathrm{m}\right)\end{array}$ & $\begin{array}{c}\tau \\
(f s)\end{array}$ & $\begin{array}{c}\mathrm{E}_{\mathrm{wwf}}^{\max }(\mathrm{GS}) \\
\left(\times 10^{11} \mathrm{~V} / \mathrm{m}\right)\end{array}$ & $\begin{array}{c}\tau \\
(f s)\end{array}$ \\
\hline 0.8 & 2.377 & 51 & 2.300 & 39 \\
\hline 1.0 & 4.543 & 45 & 4.341 & 33 \\
\hline 1.2 & 7.553 & 33 & 7.504 & 30 \\
\hline
\end{tabular}




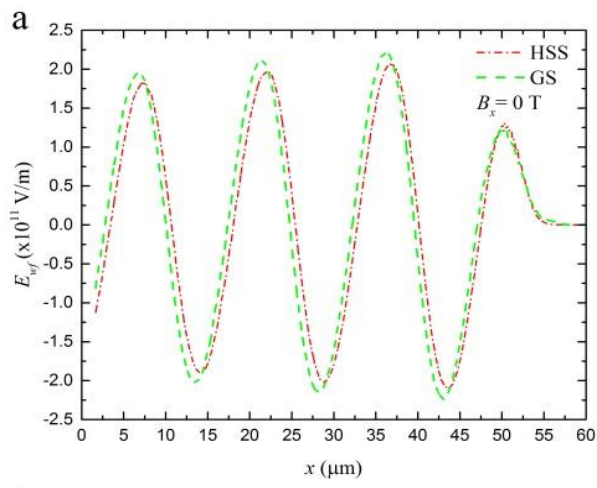

b

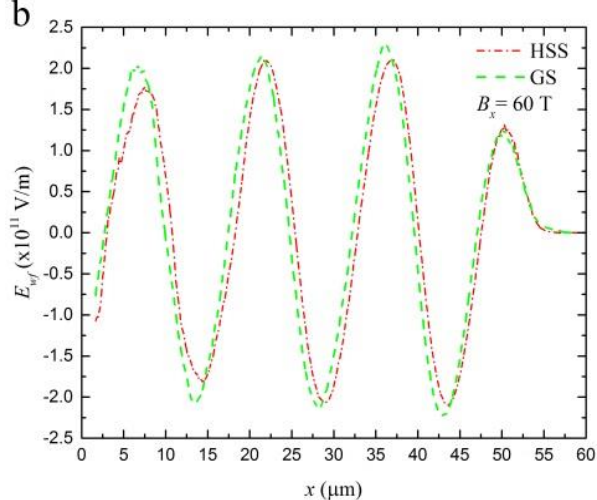

Fig. 5. Amplitude of wake field $E_{w f}$ in terms of coordinate $x$ for HSS and GS pulses with (a) $B_{x}=0 T$ and (b) $B_{x}=60 T$.
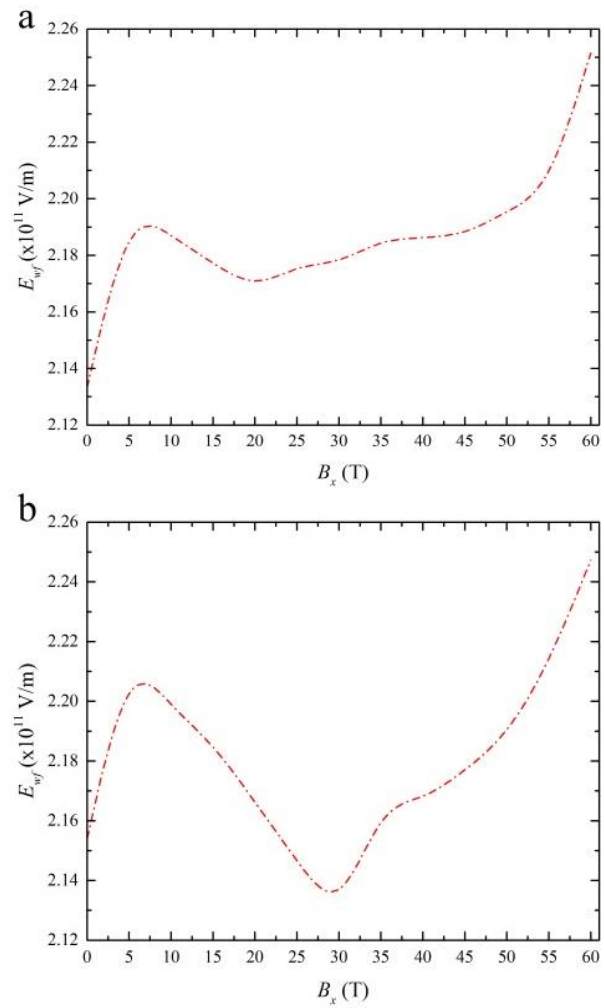

Fig. 6. Maximum amplitude of wake field $E_{w f}$ in terms of $B_{x}$ for (a) HSS pulse and (b) GS pulse.

\section{VI.EFFECT OF MAGNETIC FieLd}

Magnetic field has been a great interest in the study of laser plasma interaction. In order to have a better insight into the effect of magnetic field, the maximum amplitude of wake field in terms of $B_{x}$ is plotted for HSS and GS pulses in Figs. $6 a$ and b, respectively. It is observed that for both pulses under the mentioned conditions, applying longitudinal magnetic field causes the amplitude of the wake field peaks at $B_{x} \approx 6 T$. However, as magnetic field rises steadily, HSS and GS pulses illustrate moderate different behaviors. In HSS case, maximum $E_{w f}$ falls slightly until it reaches its lowest value at $B_{x}=20 T$, while in GS case there is a steep fall in maximum $E_{w f}$ to its lowest point, at $B_{x}=30 \mathrm{~T}$. Thereafter, HSS curve increases modestly while GS one has a dramatic growth until they both obtain their highest value at $B_{x}=60 \mathrm{~T}$. The trend of Fig. 6 for $B_{x}=0-20 T$ is the same as Figs. $5 \mathrm{a}$ and $\mathrm{b}$ in Askari and Shahidani [31]. Nevertheless, they did not study bigger values of magnetic field.

\section{Conclusion}

In this paper, we have conducted PIC simulations in order to investigate the effects of laser and plasma parameters on the wake field excitation for two different pulse shapes. For both shapes, the amplitude of wake field $E_{w f}$ grows when $n_{0}$ increases. In addition, longer laser wavelengths result in higher intensities while the pulse durations at which $E_{w f}$ peaks, fall to lower values. Comparing pulse shapes, GS excites wake field more effectively than HSS. Our study also shows the effect of applying a longitudinal magnetic field on wake field excitation. It is shown that applying appropriate magnetic field intensity enhances excitation process in all investigated cases. In other words, the magnetic strength needs to be selected correctly. Our results are compatible with the results presented by Askari and Shahidani.

\section{REFERENCES}

[1] J. Faure, C. Rechatin, O. Lundh, L. Ammoura, and V. Malka, "Injection and Acceleration of Quasimonoenergetic Relativistic Electron Beams Using Density Gradients at the Edges 
of a Plasma Channel," Phys. Plasmas, Vol. 17, pp. 083107 (1-8), 2010.

[2] S.D. Patil, M.V. Takale, V.J. Fulari, and T.S. Gill, "Sensitiveness of Light Absorption for Self-Focusing at Laser-Plasma Interaction with Weakly Relativistic and Ponderomotive Regime," J. Comput. Phys. Vol. 34, pp. 669674, 2016.

[3] P. Yu, X. Xu, V.K. Decyk, W. An, J. Vieira, F.S. Tsung, R.A. Fonseca, W. Lu, L.O. Silva, and W.B. Mori, "Modeling of Laser Wakefield Acceleration in Lorentz Boosted Frame Using Em-Pic Code with Spectral Solver," J. Comput. Phys. Vol. 266, pp. 124138, 2014.

[4] T. Tajima and J.M. Dawson, "Laser Electron Accelerator," Phys. Rev. Lett. Vol. 43, pp. 267-270, 1979.

[5] M. Litos, E. Adli, W. An, C.I. Clarke, C.E. Clayton, S. Corde, J.P. Delahaye, R.J. England, A.S. Fisher, J. Frederico, S. Gessner, S.Z. Green, M.J. Hogan, C. Joshi, W. Lu, K.A. Marsh, W.B. Mori, P. Muggli, N. Vafaei-Najafabadi, D. Walz, G. White, Z. $\mathrm{Wu}, \mathrm{V}$. Yakimenko, and G. Yocky, "HighEfficiency Acceleration of an Electron Beam in a Plasma Wakefield Accelerator," Nature, Vol. 515, pp. 92-99, 2014.

[6] D. Guénot, D. Gustas, A. Vernier, B. Beaurepaire, F. Böhle, M. Bocoum, M. Lozano, A. Jullien, R. Lopez-Martens, A. Lifschitz, and J. Faure, "Relativistic Electron Beams Driven by Khz Single-Cycle Light Pulses," Nat. Photon. Vol. 11, pp. 293-296, 2017.

[7] C.B. Schroeder, C. Benedetti, E. Esarey, M. Chen, and W.P. Leemans, "Two-Color Ionization Injection Using a Plasma Beatwave Accelerator," Nucl. Instrum. Methods Phys. Res. Vol. 909, pp. 149-152, 2018.

[8] A. Singh and N. Gupta, "Beat Wave Excitation of Electron Plasma Wave by Relativistic Cross Focusing of Cosh-Gaussian Laser Beams in Plasma," Phys. Plasmas, Vol. 22, pp. 062115 (1-10), 2015.

[9] J. Cowley, C. Thornton, C. Arran, R. J Shalloo, L. Corner, G. Cheung, C.D. Gregory, S. P. D. Mangles, N. H. Matlis, D. R. Symes, R. Walczak, and S. M. Hooker, "Excitation and Control of Plasma Wakefields by Multiple Laser Pulses," Phys. Rev. Lett. Vol. 119, pp. 044802 (1-6), 2017.
[10] S. Kumar, P.K. Gupta, R. K. Singh, S. Sharma, R. Uma, and R.P. Sharma, "PulseCompression and Self-Focusing of Gaussian Laser Pulses in Plasma Having RelativisticPonderomotive Nonlinearity," Laser Particle Beams, Vol. 35, pp. 429-436, 2017.

[11] K. Mima, J. Fuchs, T. Taguchi, J. Alvarez, J.R. Marquès, S.N. Chen, T. Tajima, and J.M. Perlado, "Self-Modulation and Anomalous Collective Scattering of Laser Produced Intense Ion Beam in Plasmas," Matter. Radiation Extremes, Vol. 3, pp. 127-134, 2018.

[12] C. Benedetti, C.B. Schroeder, E. Esarey, and W.P. Leemans, "Plasma Wakefield Excitation by Incoherent Laser Pulses: A Path Towards High-Average Power Laser-Plasma Accelerators," AIP Conf. Proc. Vol. 1777, pp. 040001 (1-6), 2016.

[13] B. Gaur, P. Rawat, and G. Purohit, "Particle Acceleration by Beating of Two Intense Cross-Focused Cosh-Gaussian Laser Beams in Plasma," Laser Particle Beams, Vol. 36, pp. 60-68, 2018.

[14] J. Faure, C. Rechatin, A. Norlin, A. Lifschitz, Y. Glinec, and V. Malka, "Controlled Injection and Acceleration of Electrons in Plasma Wakefields by Colliding Laser Pulses," Nature, Vol. 444, pp. 737-739, 2006.

[15] W.P. Leemans, B. Nagler, A.J. Gonsalves, C. Toth, K. Nakamura, C.G.R. Geddes, E. Esarey, C.B. Schroeder, and S.M. Hooker, "Gev Electron Beams from a CentimetreScale Accelerator," Nat. Phys. Vol. 2, pp. 696-699, 2006.

[16] S.P.D. Mangles, C.D. Murphy, Z. Najmudin, A.G.R. Thomas, J.L. Collier, A.E. Dangor, E. J. Divall, P.S. Foster, J.G. Gallacher, C.J. Hooker, D.A. Jaroszynski, A.J. Langley, W.B. Mori, P.A. Norreys, F.S. Tsung, R. Viskup, B.R. Walton, and K. Krushelnick, "Monoenergetic Beams of Relativistic Electrons from Intense Laser-Plasma Interactions," Nature, Vol. 431, pp. 535-538, 2004.

[17] E.A. Nanni, W.R. Huang, K-H Hong, K. Ravi, A. Fallahi, G. Moriena, R.J. Dwayne Miller, and F.X. Kärtner, "Terahertz-Driven Linear Electron Acceleration," Nat. Commun. Vol. 6, pp. 8486 (1-8), 2015.

[18] K.K. Anoop, N. Xiaochang, W. Xuan, A. Salvatore, and B. Riccardo, "Fast Ion 
Generation in Femtosecond Laser Ablation of a Metallic Target at Moderate Laser Intensity," Laser Phys. Vol. 24, pp. 105902 (1-6), 2014.

[19] Sh. Moniruzzaman, D. Lad Amit, J. Kamalesh, S. Deep, D. Indranuj, and G.R. Kumar, "Megagauss Magnetic Fields in UltraIntense Laser Generated Dense Plasmas,' Plasma Phys. Control. Fusion, Vol. 59, pp. 014007 (1-6), 2017.

[20] M. Borghesi, A.J. MacKinnon, A.R. Bell, R. Gaillard, and O. Willi, "Megagauss Magnetic Field Generation and Plasma Jet Formation on Solid Targets Irradiated by an Ultraintense Picosecond Laser Pulse," Phys. Rev. Lett. Vol. 81, pp. 112-115, 1998.

[21] R.N. Sudan, "Mechanism for the Generation of $10^{9} \mathrm{G}$ Magnetic Fields in the Interaction of Ultraintense Short Laser Pulse with an Overdense Plasma Target," Phys. Rev. Lett. Vol. 70, pp. 3075-3078, 1993.

[22] A. Lagutin, K. Rosseel, F. Herlach, J. Vanacken, and Y. Bruynseraede, "Development of Reliable 70 T Pulsed Magnets," Meas. Sci. Technol. Vol. 14, pp. 2144-2150, 2003.

[23] P.K. Shukla, G. Brodin, M. Marklund, and L. Stenflo, "Wake Field Generation and Nonlinear Evolution in a Magnetized Electron-Positron-Ion Plasma," Phys. Plasmas, Vol. 15, pp. 082305 (1-9), 2008.

[24] V.B. Krasovitskii, V.G. Dorofeenko, V.I. Sotnikov, and B.S. Bauer, "Interaction of Powerful Laser Pulse with Magnetized Plasma," Phys. Plasmas, Vol. 11, pp. 724-742, 2004.

[25] C.K. Birdsall and A.B. Langdon, Plasma Physics Via Computer Simulation. McGrawHill, 1985

[26] H. Chun Wu, Jpic and How to Make a Pic Code, Cornell University Library, arXiv.org, 2011,

[27] E. Esarey and C.B. Schroeder, "Physics of Laser-Driven Plasma-Based Acceleration," Vol., pp. 53510 (1-40), 2003,

[28] C. Huang, V.K. Decyk, C. Ren, M. Zhou, W. Lu, W.B. Mori, J.H. Cooley, Jr.T.M.
Antonsen, and T. Katsouleas, "Quickpic: A Highly Efficient Particle-in-Cell Code for Modeling Wakefield Acceleration in Plasmas," J. Comput. Phys. Vol. 217, pp. 658679, 2006.

[29] K. Yee, "Numerical Solution of Initial Boundary Value Problems Involving Maxwell's Equations in Isotropic Media," IEEE Trans. Antennas Propag. Vol. 14, pp. 302-307, 1966.

[30] J.P. Boris, "Relativistic Plasma SimulationOptimization of a Hybrid Code," Proceeding of Fourth Conference on Numerical Simulations of Plasmas, 1970.

[31] H.R. Askari and A. Shahidani, "Influence of Properties of the Gaussian Laser Pulse and Magnetic Field on the Electron Acceleration in Laser-Plasma Interactions," Opt. Laser Technol. Vol. 45, pp. 613-619, 2013.

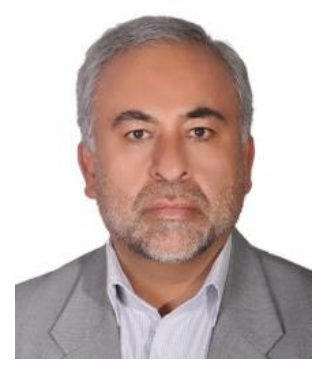

Hossein Zahed, $\mathrm{PhD}$ in plasma physics, Associate Professor at Sahand University of Technology, Tabriz. Iran.

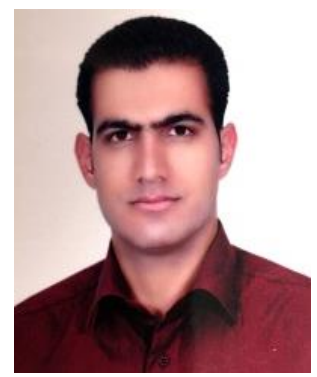

Amir Rahimian received his M.Sc. degree in plasma physics from Sahand University of Technology in 2009. He is currently a Faculty member of Qeshm Azad University. He is a member of Physics Society of Iran. 